ISSN 1997-5902

\title{
Production et retombées minérales des litières chez quatre espèces de légumineuses arborées, utilisées en amélioration de jachères, en zone forestière de Côte d'Ivoire
}

\author{
*GNAHOUA Guy Modeste', OLIVER Robert'2, NGUESSAN Kanga Anatole', BALLE Pity ${ }^{3}$ \\ ${ }^{1}$ Centre National de Recherche Agronomique 08 B.P. 801 Abidjan 08. Côte d'Ivoire \\ ${ }^{2}$ Centre de Coopération Internationale en Recherche Agronomique pour le Développement (CIRAD). 34.000 Montpellier, \\ France. \\ ${ }^{3}$ Centre de Recherche en Écologie 08 B.P. 109 Abidjan 08. Côte d'Ivoire \\ *Auteur correspondant : gm.gnahoua@hotmail.fr
}

Original submitted in on 20th July 2013 Published online at www.m.elewa.org on 30 th December 2013. https://dx.doi.org/10.4314/jab.v72i1.99665

\section{RÉSUMÉ}

Objectifs : Ce travail étudie les aptitudes des arbres fixateurs d'azote à restaurer la fertilité des sols dans les pratiques agroforestières d'amélioration des jachères. De façon spécifique, la présente étude vise à quantifier les productions de biomasse foliaire afin d'évaluer la restitution des éléments minéraux dans de tels systèmes.

Méthodologie et Résultats : Les litières produites par les arbres ont été collectées et leur composition minérale $(\mathrm{N}, \mathrm{P}$ et $\mathrm{K})$ a été déterminée. Les résultats ont montré une variation saisonnière de la production de litière. Leucaena leucocephala a montré les productions de litière et la teneur en azote les plus élevées (8,8 tha $^{-1}$ et $\left.3,2 \%\right)$. La teneur en azote des litières restée stable toute l'année semble confirmer le phénomène de la remobilisation de cet élément par la plante. Les composés phosphoriques et potassiques étaient très faibles.

Conclusion et application : Les espèces étudiées, âgées seulement de 5,5 ans ont montré une capacité de production de litières et de libération d'éléments minéraux aussi importante que celles des formations naturelles utilisées traditionnellement comme jachère. La chute des litières est une phase importante dans le cycle biogéochimique des éléments nutritifs qui assurent la productivité des écosystèmes naturels et cultivés. La connaissance des quantités d'éléments minéraux libérés et de la vitesse de recyclage de ceux-ci permet de faire un bilan de leur utilisation par les plantes et d'en déterminer les excès ou les carences dans un écosystème donné. Par les dépôts d'abondantes biomasses au sol et les quantités d'azote qu'elles peuvent libérer, les espèces étudiées se sont montrées prometteuses pour les pratiques agroforestières d'amélioration des jachères.

Mots clés: légumineuses arborées, amélioration de jachère, chute de litière, nutriments, agroforesterie 


\section{ABSTRACT}

Production and mineral impact of litter from leguminous trees used to improve fallow land in the forest zone of Côte d'Ivoire

Objective: This study aims at assessing the performance of nitrogen-fixing trees to restore soil fertility in fallow land for improvement practices. The current study aims to quantify the production of leaf biomass and the amount of nutrients released in such agroforestry systems.

Methodology and Results: Leaf litter production was monitored under four legume tree species: Acacia auriculiformis, Acacia mangium, Albizia lebbeck and Leucaena leucocephala. The leaf litter nutrient contents (N, P and K) were determined by laboratory analysis. Results showed a seasonal variation in litter production; dry seasons being the periods of highest litter fall. Leucaena leucocephala had the highest litter production and nitrogen concentration (8.8 t.ha-1. $\mathrm{y}^{-1}$ and $3.2 \%$ ). Monthly nitrogen concentrations remained stable throughout the year in the litter, indicating possible relocation of nitrogen to other parts of trees before the leaves fall. Phosphorus and potassium concentrations were very low and reflected local soil deficiencies.

Conclusion and application: The tree species studied were only 5.5 years and showed litter production and Nitrogen release capacities that were as important as those of natural vegetations traditionally used as fallows. Litter fall is an important step in the biogeochemical cycling of nutrients that ensure the productivity of natural and cultivated ecosystems. Thus, knowledge about nutrients amounts and their recycling delays are key tools to assess nutrient utilization by plants and to determine their excess or deficiencies in a given ecosystem. Through the abundant deposits of leaf biomass on ground and the amount of nitrogen they can rapidly release, the species studied, showed good prospects for fallow land improvement practices.

Keywords: leguminous trees, improved fallow, litter fall, nutrients, agroforestry

\section{INTRODUCTION}

Dans les systèmes traditionnels de cultures itinérantes sur brûlis des zones tropicales, la jachère naturelle longue de $10-30$ ans qui permettait autrefois de restaurer la fertilité des sols s'est raréfiée ; les sols sont remis en cultures, après 3 à 5 ans de jachères, alors que leurs capacités productives ne sont pas restaurées (Floret et Pontanier, 1999 ; Jouve, 2001). Les conséquences de cette situation sont la baisse de la fertilité des sols, des rendements, l'érosion, la pression des adventices et la raréfaction des produits de prélèvement autrefois abondants dans les jachères (bois de feu, aliments et médicaments). Face à cette dégradation de l'environnement agroécologique et la nécessité de pérenniser les productions agricoles en Afrique subsaharienne, la recherche de systèmes alternatifs à l'agriculture itinérante a abouti à l'exploration des techniques agroforestières d'amélioration des jachères (Peltier et Eyog-Matig,
1988). L'amélioration des jachères consiste à substituer à la jachère naturelle longue, des jachères courtes d'arbres fixateurs d'azote (les légumineuses généralement). Grâce à leur croissance rapide, ces espèces accélèrent les processus biologiques et physico-chimiques de restauration de la fertilité des sols (Moraud, 1993) et réduisent la viabilité du stock séminal des adventices (De Rouw, 1993). Les espèces fixatrices d'azote peuvent se développer sur des sols appauvris par des cycles successifs de cultures ; par leurs racines qui explorent des horizons profonds du sol, ces arbres permettent le recyclage des éléments minéraux lixiviés sous les cultures et même ceux issus de la roche mère en décomposition (Moraud, op.cit). L'azote fixé pour leur métabolisme est ensuite restitué au sol par l'intermédiaire de la litière foliaire, les exsudats et nécromasses racinaires. Les légumineuses arborées sont présentées comme prometteuses 
pour l'amélioration de la nutrition azotée des cultures associées (De Tafin et al., 1991) et la production de bois d'utilisation courante (BernhardReversat et al., 1993 ; Dommergues et al., 1999). Introduits en basse Côte d'lvoire au début de la décennie 1980, les acacias australiens (Acacia mangium et $A$. auriculiformis) ont fait l'objet d'études sylvicoles (Dupuy et Nguessan, 1991) et

\section{MATERIEL ET METHODES}

Site de l'étude : L'étude a eu pour cadre la Station du Centre National de Recherche Agronomique (CNRA) à Oumé ( $06^{\circ} 17 \mathrm{LN}$ et $05^{\circ} 31 \mathrm{LO}$ ), en région Centre Ouest de Côte d'Ivoire. Selon Clément (1970), le climat de la région est subéquatorial avec deux saisons de pluies (mars-juin et août-octobre) et deux saisons sèches (juillet et novembre-février). La température moyenne annuelle est de $26,5^{\circ} \mathrm{C}$ et la pluviométrie de $1350 \mathrm{~mm}$ par an. Les sols sont ferralitiques moyennement désaturés et globalement bien drainés. La végétation d'origine est une forêt dense humide semi décidue.

Matériel végétal : L'étude a porté sur quatre légumineuses arborées de la famille des Mimosacées (Figure 1) : Acacia mangium Will., Acacia auriculiformis A. Cunn., Albizia lebbeck (L) Benth. et Leucaena glauca Willd. Les deux acacias sont d'origine australienne. Les deux autres espèces sont sub-spontanées et considérées d'associations avec les cocotiers sur les sables quaternaires du littoral (Dupuy et Nguessan, 1990). Cependant, ces légumineuses exotiques et celles bien connues localement n'avaient pas encore été étudiées au niveau des aptitudes à restaurer la fertilité des sols agricoles dégradés par le raccourcissement de la durée des jachères.

comme locales. II s'agissait donc d'étudier, comparativement, des espèces exotiques en cours d'introduction et celles déjà adaptées aux conditions écologiques locales.

Dispositif expérimental : L'essai d'amélioration des jachères a été implanté sur une parcelle précédemment cultivée en vivriers (igname, manioc) et colonisée par Chromolaena odorota, après abandon. Le dispositif expérimental est un bloc de Fisher avec 3 répétitions et 5 traitements. Les traitements correspondent aux 4 végétations de jachères améliorées : Acacia auriculiformis, Acacia mangium, Albizia lebbeck, Leucaena leucocephala et à la jachère naturelle dominée par Chromolaena odorata. Les légumineuses ont été plantées à $2 \mathrm{~m} \times 2 \mathrm{~m}$, soit 2500 arbres.ha- $^{-1}$. Chaque parcelle unitaire mesure $10 \mathrm{~m} \times 10 \mathrm{~m}\left(100 \mathrm{~m}^{2}\right)$ et comporte 36 arbres.

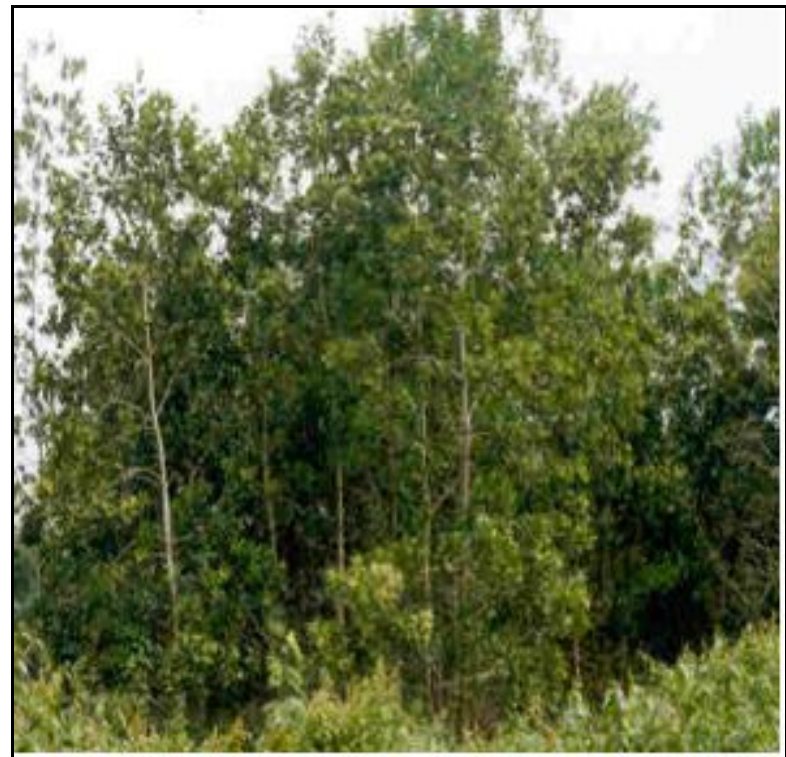

A. Jachère améliorée d'Acacia auriculiformis

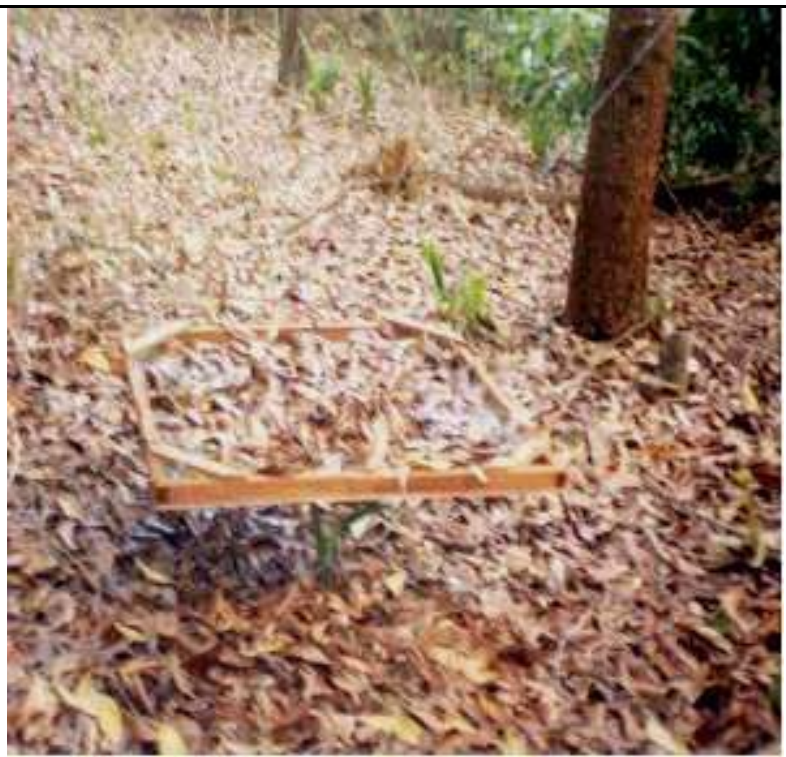

B. Dépôt de litière dans une jachère d'Acacia mangium 


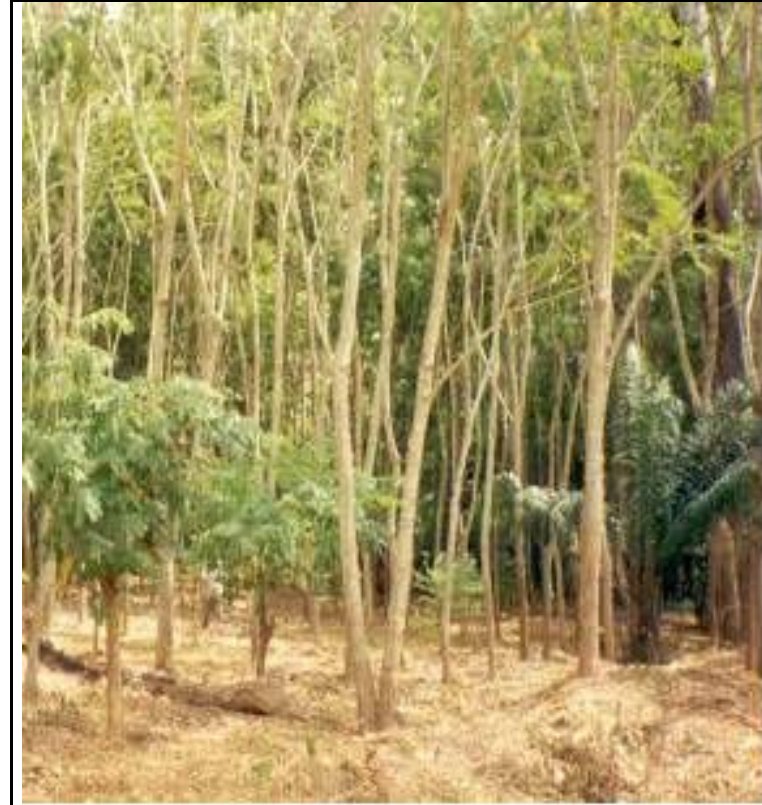

C. Jachère d'Albizia lebbeck avec régénération

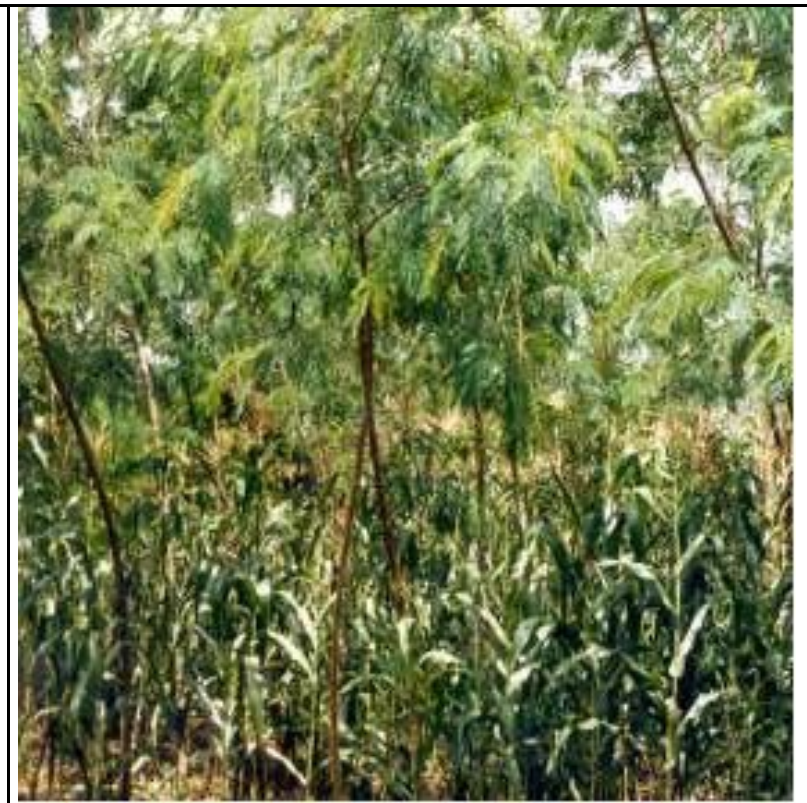

D. Leucaena leucocephala en association avec le maïs

Figure 1 : Espèces légumineuses arborées étudiées dans le dispositif d'amélioration de jachères

\section{Caractérisation des litières}

1. Suivi de la chute des litières : La production de litière a été suivie chez les 4 espèces de légumineuses seulement. La jachère naturelle à Chromolaena odorata a posé quelques problèmes méthodologiques pour la collecte des litières en chute. Dans les 4 peuplements arborées âgés de 5 ans, la biomasse aérienne en chute (feuilles, fleurs, fruits, brindilles) a été collectée à intervalle de 15 jours pendant une année entière. Pour ce faire, nous avons installé dans le carré central de chaque parcelle unitaire un collecteur de litière constitué par une toile moustiquaire de $1 \mathrm{~m}$ de côté montée sur un cadre en bois et maintenu à environ $20 \mathrm{~cm}$ du sol. Lors des prélèvements périodiques des litières, la biomasse foliaire a été séparée des brindilles et des gousses. Pour caractériser la dynamique de la chute des litières à l'échelle de l'année, nous avons procédé à la sommation mensuelle des poids de la biomasse foliaire collectée chaque 15 jours.

2. Litières présentes au sol à la fin de la phase de jachère : Les litières présentes au sol ont été

\section{RESULTATS}

Quantités de litières collectées et teneur en azote : Les quantités totales de litières produites par les peuplements étudiés sont présentées au tableau 1. Les quantités de biomasse de Leucaena leucocephala $(8,8$ ramassées dans des carrés de $1 \mathrm{~m}$ de côté dans les parcelles âgées de 6 ans, à la fin de la période de jachère. Lorsque les quantités de litières qui tombent annuellement sont connues, l'intérêt de la quantification des litières présentes au sol à la fin de la phase de jachère est de déterminer la vitesse de décomposition de celles-ci.

Quantification des litières et compositions minérales : Les litières ont été ont séchées à l'étuve à $70^{\circ} \mathrm{C}$ pendant 24 heures en vue de la détermination des poids sec de résidus produits par espèce. L'analyse des teneurs en azote total a été faite sur un " auto analyseur TECHNICON ». Les autres éléments ( $P$ et $K$ ) ont été dosés par la méthode ICP (spectrométrie d'absorption atomique à lampe plasma).

Analyse des données : Les données mesurées ont fait l'objet de saisies à l'aide du logiciel EXCEL 2007 et d'analyses de variance au seuil de signification de $5 \%$, à l'aide du logiciel STATISTICA version 7.1. En cas de différences significatives, le test de Newman-Keuls a été utilisé pour constituer les groupes homogènes.

tha $\left.{ }^{-1} \mathrm{an}^{-1}\right)$ ont été supérieures $(P<0,05)$ à celles des deux Acacias et Albizia lebbeck (qui ont affiché des productions statistiquement identiques (7,3 à 7,8 tha$\left.{ }^{1} \mathrm{an}^{-1}\right)$. Les concentrations en azote des litières ont varié 
entre 1,51 et 3,13\% (Tableau 1). Albizia lebbeck et Leucaena leucocephala ont montré les teneurs les plus élevées $(3,13$ et $3,02 \%$ respectivement) alors que Acacia auriculiformis et $A$. mangium ont affiché des concentrations plus faibles $(1,63$ et $1,65 \%$, respectivement). Ces résultats ont montré la relative pauvreté en azote des litières des deux acacias.

Tableau 1 : Quantités de litières collectées pendant une année (t.ha- ${ }^{-1} \mathrm{an}^{-1}$.) et leur teneur en azote $(\%)$

\begin{tabular}{lcccc}
\hline Espèces & $\begin{array}{c}\text { Acacia } \\
\text { auriculiformis }\end{array}$ & $\begin{array}{c}\text { Acacia } \\
\text { mangium }\end{array}$ & $\begin{array}{c}\text { Albizia } \\
\text { lebbeck }\end{array}$ & $\begin{array}{c}\text { Leucaena } \\
\text { leucocephala }\end{array}$ \\
\hline Quantité de litière (tha) & $7,3(\mathrm{~b})$ & $7,8(\mathrm{~b})$ & $7,4(\mathrm{~b})$ & $8,8(\mathrm{a})$ \\
$\mathrm{N}(\%)$ & $1,63 \mathrm{~b}$ & $1,651 \mathrm{~b}$ & $3,13 \mathrm{a}$ & $3,02 \mathrm{a}$ \\
\hline
\end{tabular}

Deux moyennes affectées de la même lettre ne diffèrent pas par le test de Newman-Keuls

Composition minérale des litières collectées : Les compositions minérales des litières ont montré des différences assez importantes entre les espèces étudiées (Tableau 2). D'une manière générale, Albizia lebbeck et Leucaena leucocephala ont libéré plus d'éléments fertilisants que les deux Acacias australiens. Leucaena leucocephala a affiché les apports les plus élevés d'éléments fertilisants $(P<0,05)$ : azote $\left(266 \mathrm{~kg} \cdot \mathrm{ha}^{-1}\right)$, phosphore (22,91 kg.ha-1) et potassium (208,45 kg.ha-1). Acacia auriculiformis a fourni au sol les quantités les plus faibles d'azote (119 kg.ha-1), de phosphore $\left(4,58 \mathrm{~kg}^{-h^{-1}}{ }^{-1}\right)$ et de potassium $\left(11,81 \mathrm{~kg} \cdot \mathrm{ha}^{-1}\right)$. Globalement, les apports de phosphore et de potassium des feuilles ont été relativement faibles et reflètent la déficience des sols de la zone d'étude.

Tableau 2 : Retombées minérales annuelles des litières collectées (kg.ha-1) sous les 4 espèces

\begin{tabular}{lcccc}
\hline Éléments & $\begin{array}{c}\text { Acacia } \\
\text { auriculiformis }\end{array}$ & $\begin{array}{c}\text { Acacia } \\
\text { mangium }\end{array}$ & $\begin{array}{c}\text { Albizia } \\
\text { lebbeck }\end{array}$ & $\begin{array}{c}\text { Leucaena } \\
\text { leucocephala }\end{array}$ \\
\hline $\mathrm{N}$ & $119 \mathrm{~b}$ & $129 \mathrm{~b}$ & $232 \mathrm{a}$ & $266 \mathrm{a}$ \\
$\mathrm{P}_{2} \mathrm{O}_{5}$ & $4,58 \mathrm{~b}$ & $4,58 \mathrm{~b}$ & $20,62 \mathrm{a}$ & $22,91 \mathrm{a}$ \\
$\mathrm{K}_{2} \mathrm{O}$ & $11,81 \mathrm{~d}$ & $85,55 \mathrm{c}$ & $115,68 \mathrm{~b}$ & $208,45 \mathrm{a}$ \\
\hline
\end{tabular}

Deux moyennes affectées de la même lettre ne diffèrent pas par le test de Newman-Keuls au seuil de $5 \%$.

Dynamique de la chute des litières Pour toutes les espèces étudiées, la production de litière à l'échelle de l'année a suivi à peu près la même dynamique (Figure 2). On a distingué globalement :

- deux phases de diminution des quantités de litière, de février à mai d'une part, et d'août à novembre d'autre part, correspondant aux périodes pluvieuses ;
- deux phases d'augmentation de la production allant de juin à août puis de novembre à janvier, qui se situent respectivement dans la petite et la grande saison sèche.

- $\quad$ le mois de juillet est celui des plus importantes productions de litières pour toutes les espèces. 


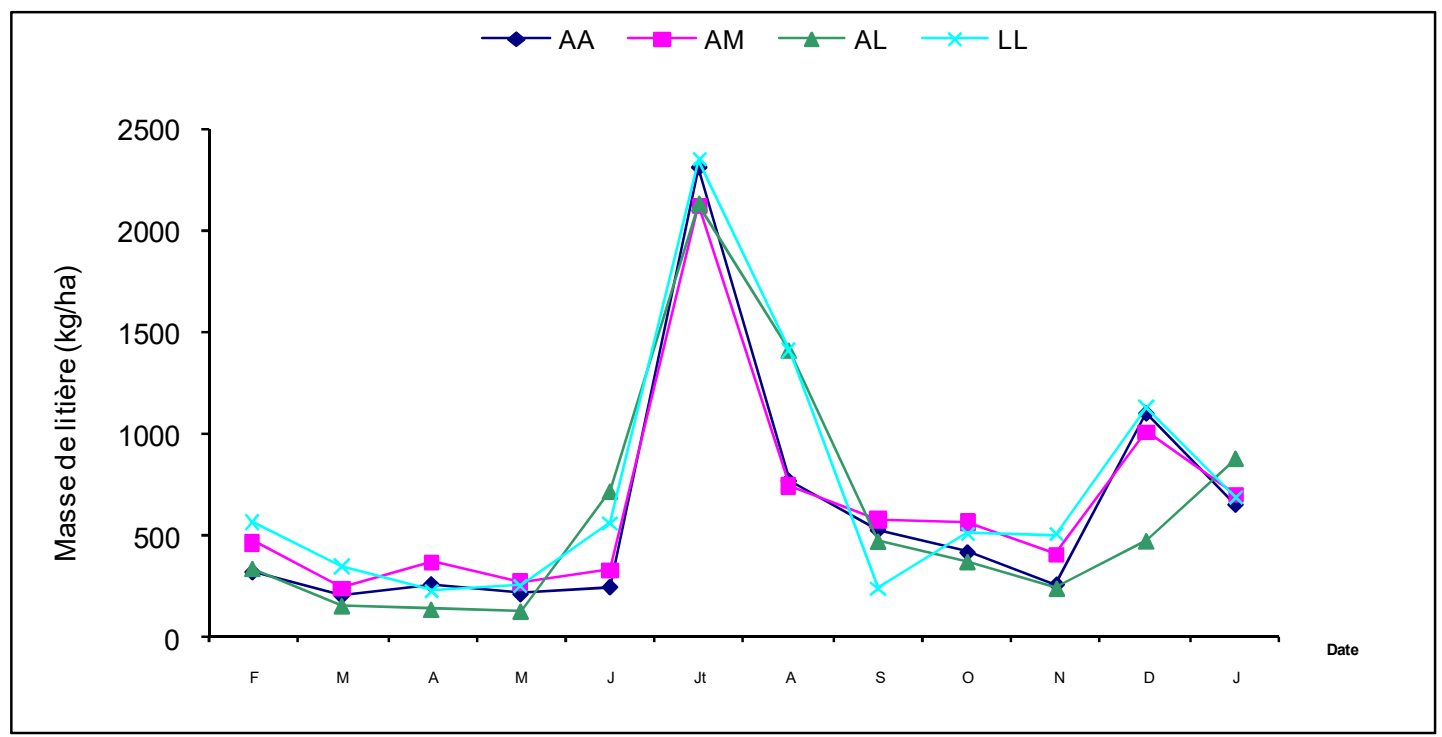

Figure 2 : Dynamique annuelle de la production de litière dans les 4 peuplements étudiés

Dynamique des retombées azotées des litières Les retombées azotées étant les plus significatives, leur dynamique a été suivie au cours de l'année. Les courbes des retombées d'azote (figure 3 ) ont montré des allures identiques à celles de la chute des litières. Comme dans le cas précédent, la paire Albizia lebbeckLeucaena leucocephala et celle formée par Acacia auriculiformis-Acacia mangium ont eu des évolutions parallèles tout au long de l'année. Ces courbes d'évolution ont montré deux périodes d'augmentation des retombées azotées ; l'une en juillet (petite saison sèche) qui correspond aux retombées d'azote les plus élevées et l'autre en novembre (début de la grande saison sèche). Deux autres périodes de diminution des retombées azotées ont été notées ; il s'agit respectivement des débuts de la grande saison et de la petite saison des pluies (mars et septembre).

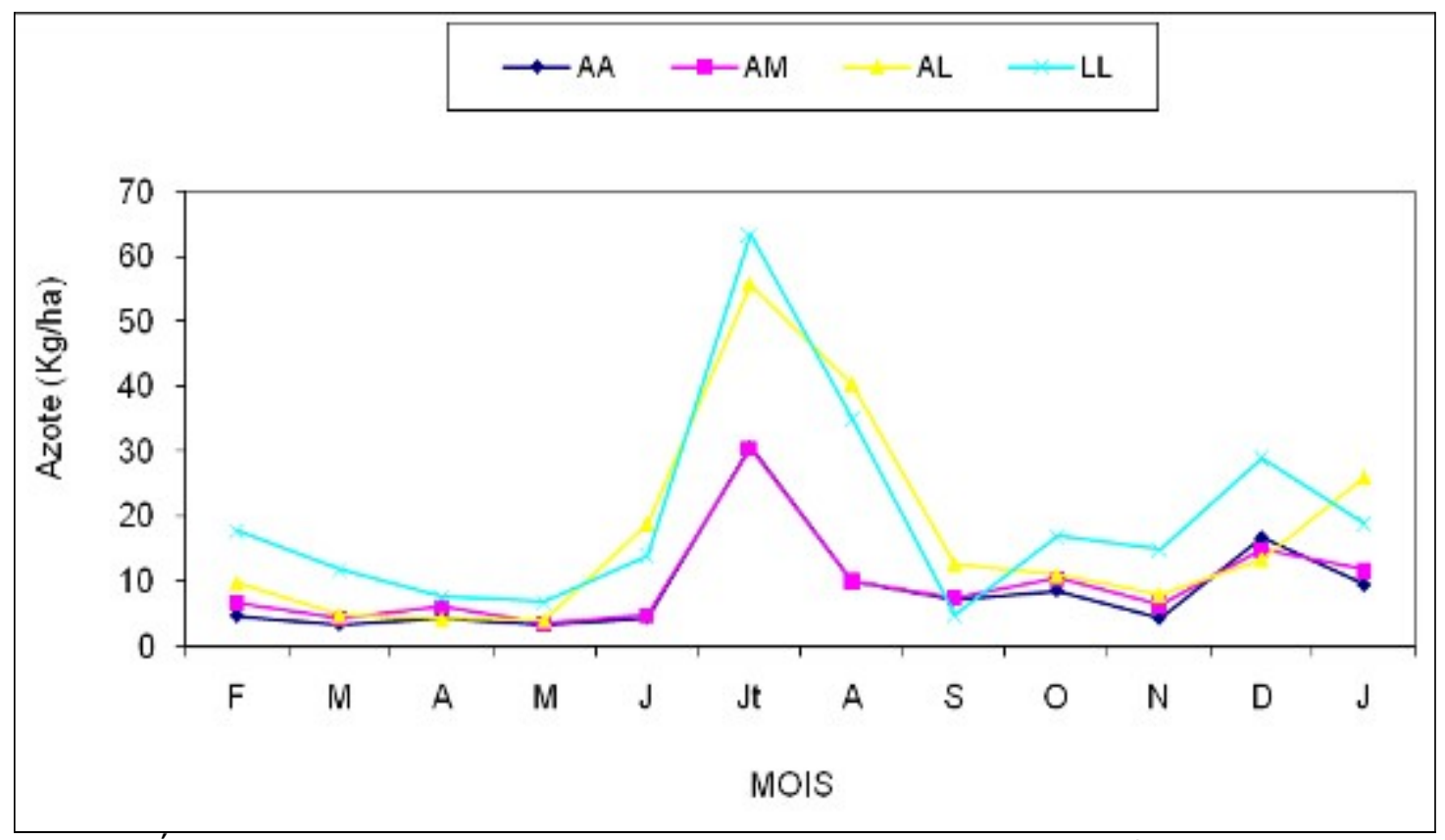

Figure 3 : Évolution annuelle des retombées azotées des litières collectées dans les 4 peuplements étudiés 
Les litières présentes au sol à la fin de la phase de jachère arborée : Le tableau 3 montre les quantités de litières présentes au sol à la fin de la phase arborée. L'espèce Acacia mangium a montré les quantités de litières les plus importantes $\left(10,9\right.$ tha $\left.^{-1}\right)$ tandis que les plus faibles quantités $(3,6$ tha-1) ont été enregistrées sous Leucaena leucocephala. Les résidus de Leucaena leucocephala étaient composés essentiellement de brindilles et de rachis. Les litières présentes au sol sous Leucaena leucocephala étaient deux à trois fois inférieures à celles observées sous Acacia mangium, Acacia auriculiformis et Albizia lebbeck. Pour ces trois dernières espèces, les quantités de litières trouvées au sol (8,1 à 10,9 tha-1) étaient supérieures à celles collectées précédemment en une année $\left(7,3\right.$ à 8,8 tha- $\left.{ }^{-1}\right)$.

Tableau 3 : Quantités de litière présentes au sol (t.ha-1) en fin de jachère et teneurs en azote $(\%)$

\begin{tabular}{lcccc}
\hline Espèces & $\begin{array}{c}\text { Acacia } \\
\text { mangium }\end{array}$ & $\begin{array}{c}\text { Acacia } \\
\text { auriculiformis }\end{array}$ & $\begin{array}{c}\text { Albizia } \\
\text { lebbeck }\end{array}$ & $\begin{array}{c}\text { Leucaena } \\
\text { leucocephala }\end{array}$ \\
\hline Quantité de litière & $10,9 \mathrm{a}$ & $9,2 \mathrm{ab}$ & $8,1 \mathrm{~b}$ & $3,6 \mathrm{c}$ \\
$\mathrm{N} \%$ & $1,51 \mathrm{ab}$ & $1,40 \mathrm{~b}$ & $1,84 \mathrm{a}$ & $1,30 \mathrm{~b}$ \\
\hline
\end{tabular}

Deux moyennes affectées de la même lettre ne diffèrent pas par le test de Newman-Keuls à $P=0,05$

La teneur en éléments fertilisants des litières a présenté des différences significatives à très hautement significatives (tableau 4). La teneur en azote la plus élevée était celle des résidus d'Albizia lebbeck (1,84\%). A. mangium a présenté la teneur la plus faible $(1,404 \%)$. La teneur moyenne en azote des litières présentes au sol est en légère baisse comparativement à celle des litières en chute. Les teneurs, en ce qui concerne le phosphore et le potassium, étaient très faibles comme dans le cas des litières collectées. Concernant le phosphore, les plus faibles concentrations en $\mathrm{P}_{2} \mathrm{O}_{5}$ ont été trouvées dans les litières de Leucaena leucocephala $(0,075 \%)$ et de $A$. mangium $(0,0594 \%)$. Les teneurs les plus fortes ont été trouvées chez $A$. auriculiformis $(0,183 \%)$. Dans le cas du potassium, on a noté une teneur très élevée $(3,00 \%)$ dans les feuilles de $L$. leucocephala alors que la concentration chez les trois autres légumineuses oscillait entre 0,349 et $0,902 \%$. Concernant les retombées minérales de ces litières (Tableau 4), celles de l'azote ont varié entre $47 \mathrm{~kg} \mathrm{ha}^{-1}$ (L. leucocephala) et $153 \mathrm{~kg} \cdot \mathrm{ha}^{-1}(A$. mangium). Les retombées les plus importantes ont été obtenues pour le phosphore dans les litières d'A. auriculiformis $\left(16,861 \mathrm{~kg}^{-h a^{-1}}\right)$ et pour le potassium dans celles de L. leucocephala $\left(108,189 \mathrm{~kg}^{-h^{-1}}\right)^{-1}$.

Tableau 4: Retombées minérales des litières trouvées au sol en fin de jachère (kg.ha-1)

\begin{tabular}{lcccc}
\hline Élément & $\begin{array}{c}\text { Acacia } \\
\text { auriculiformis }\end{array}$ & $\begin{array}{c}\text { Acacia } \\
\text { mangium }\end{array}$ & $\begin{array}{c}\text { Albizia } \\
\text { lebbeck }\end{array}$ & $\begin{array}{c}\text { Leucaena } \\
\text { leucocephala }\end{array}$ \\
\hline $\mathrm{N}$ & $139 \mathrm{a}$ & $153 \mathrm{a}$ & $149 \mathrm{a}$ & $47 \mathrm{~b}$ \\
$\mathrm{P}_{2} \mathrm{O}_{5}$ & $16,861 \mathrm{a}$ & $6,83 \mathrm{c}$ & $12,80 \mathrm{~b}$ & $2,721 \mathrm{~d}$ \\
$\mathrm{~K}_{2} \mathrm{O}$ & $83,034 \mathrm{~b}$ & $85,11 \mathrm{~b}$ & $28,305 \mathrm{c}$ & $108,189 \mathrm{a}$ \\
\hline
\end{tabular}

Deux moyennes affectées de la même lettre ne diffèrent pas par le test de Newman-Keuls à $P=0,05$

\section{DISCUSSION}

La production de litière de la présente étude $(7,3$ à 8,8 t.ha-1.an-1) en zone forestière, s'est montrée supérieure aux valeurs de 5,1 t.ha- $^{-1}$.an-1 enregistrées par Zakra (1997) à Port-Bouët (sur le littoral ivoirien) dans un peuplement d'Acacia spp de cinq (5) ans planté à $4 \mathrm{~m} \mathrm{x}$ $4 \mathrm{~m}$. Elle est également supérieure aux quantités de 6,1 et 6,4 tha-1. an $^{-1}$ observées par Gona-Tchimbakala et al.(2005) qui ont étudié la chute annuelle de litière dans des peuplements de Fraké (Terminalia superba) de 7 et 12 ans au Congo. Une telle production de biomasse à 5,5 ans seulement résulte de la croissance rapide de ces légumineuses. Les pics de production de litières qui se situent exclusivement dans les périodes sèches montrent que la chute des feuilles est liée aux variations climatiques. Bien qu'il soit reconnu qu'en zone tropicale, le stress hydrique prononcé en saison sèche 
soit responsable de la forte chute des feuilles, certains travaux rapportent des cas de productions de litière plus importantes en saison pluvieuse (Gona-Tchimbakala et al.2005). Le faible niveau des concentrations en azote des litières d'acacias $(1,6 \%)$ est également observé par Agbahungba (2007) qui rapporte des valeurs identiques (moins de 2\%) dans les litières d'Acacias plantés au Sud Bénin. La similitude des courbes d'évolution annuelle de la production de litières et des retombées azotées suggère que les concentrations en azote des litières sont restées constantes toute l'année. Ces observations semblent confirmer l'hypothèse de la remobilisation de l'azote vers d'autres parties de la plante avant la chute des feuilles sénescentes (Aerts, 1996 ; Chiharu et al, 2007).

Toutefois, la baisse prononcée de la teneur en azote observée chez Leucaena leucocephala en septembre pourrait être le fait du pluviolessivage. Dans le cas particulier des litières d'acacias dont les teneurs en azote sont les plus faibles (1,6\%), Gutteridge (1992) et Zakra (1997) ont évoqué la présence dans leurs feuilles d'abondants composés organiques (celluloses, hémicelluloses, lignine, phénols et tanins). Malgré tout, les retombées en azote de ces légumineuses (119 à $266 \mathrm{~kg} \mathrm{ha}^{-1} \mathrm{an}^{-1}$ ) se sont montrées suffisantes et pouvaient entretenir, pour certaines, plusieurs cycles successifs de cultures vivrières (Gnahoua, 2004).

Le faible niveau des retombées en phosphore et en potassium reflète en partie les carences naturelles des sols de Oumé mais il peut également résulter d'une immobilisation plus forte de ces éléments dans les feuilles et les parties lignifiées des arbres. Le phosphore est après l'azote la seconde carence des sols ferrallitiques selon Boyer (1982) et dans le cas précis de la région d'Oumé en zone forestière de Côte d'Ivoire, les teneurs moyennes signalées par Oualou (1989) sont de 0,48\%, ce qui indique que ces sols sont très pauvres en cet élément. Comparée aux chutes annuelles de litières $\left(7,3\right.$ à $8,8 \mathrm{t} \cdot$ ha $^{-1}$.an-1 $\left.{ }^{-1}\right)$, la biomasse

\section{CONCLUSION}

Les quantités de litières produites annuellement par les espèces étudiées sont élevées et sont capables de restituer rapidement au sol d'importantes retombées minérales, notamment l'azote. Cependant, les retombées phosphoriques et potassiques des litières peuvent être assez faibles dans ce genre de système et nécessiter des apports extérieurs de fertilisants pour suppléer ces carences en cas de mise en culture de ces sols. La production de litière a mis en évidence Leucaena présente au sol en fin de jachère a montré une tendance à l'accumulation pour Acacia auriculiformis, Acacia mangium et Albizia lebbeck (respectivement : 10,9, 9,2 et 8,1 $\mathrm{t}_{\text {ha- }}{ }^{-1}$ ), indiquant ainsi que les résidus produits en une année par ces espèces ne se décomposent pas tous immédiatement. A l'inverse, chez Leucaena leucocephala, les résidus au sol (3,6 t.ha-1) étaient plus faibles en quantités que ceux tombant en une année $\left(8,8\right.$ tha $\left.^{-1} \cdot \mathrm{an}^{-1}\right)$. En outre, les concentrations en azote des litières présentes au sol étaient plus faibles que celles des litières en chute, conséquence du processus de décomposition et de libération des éléments minéraux qui affecte les résidus au sol. On passe en effet pour les acacias australiens à des concentrations moyennes de 1,6 à $1,4 \%$ tandis chez les deux autres légumineuses, la diminution est plus forte. De $3 \%$ et plus, les teneurs diminuent à 1,84 et $1,30 \%$ indiquant une minéralisation plus rapide de l'azote chez ces espèces. Contrairement aux phyllodes coriaces des acacias australiens qui peuvent atteindre 10 à $15 \mathrm{~cm}$ de longueur et $8 \mathrm{~cm}$ de largeur, les feuilles de Leucaena leucocephala et Albizia lebbeck sont composées de folioles de très faibles dimensions (moins de $2 \mathrm{~cm}$ de diamètre). Dans une étude sur la biodégradabilité des litières collectées à Oumé, Oliver et Ganry (1994) ont noté que les feuilles des acacias ont été plus résistantes à la dégradation que celles de Leucaena leucocephala et d'Albizzia lebbeck. II avait en outre déterminé des rapports $\mathrm{C} / \mathrm{N}$ de 18 et $21 \mathrm{chez}$ Acacia mangium et Acacia auriculiformis, respectivement contre 12 et 13 pour Albizzia lebbeck et Leucaena leucocephala. . Les deux acacias australiens, quoique plus abondamment utilisés dans les dispositifs d'agroforesterie en Côte d'Ivoire (Ballé et Nklo, 1986 ; De Tafin et al., 1991 ; Dupuy et Nguessan, 1990) ont montré des performances limitées pour des espèces considérées comme ayant un haut potentiel de fixation d'azote (NAS, 1986 ; Schroth et al.,1995).

leucocephala et Acacia mangium tandis que les retombées azotées ont montré les performances de Leucaena leucocephala et Albizia lebbeck. La chute des litières est une phase importante dans le cycle biogéochimique des éléments nutritifs qui assurent la productivité des écosystèmes naturels et cultivés. La connaissance des quantités de biomasse qui retournent au sol et des minéraux libérés permet avec d'autres critères comme la biomasse et la vitesse de 
décomposition de faire un bilan de leur utilisation par les plantes et d'en déterminer les excès ou les carences dans un écosystème donné. Par les dépôts d'abondantes biomasses au sol et les quantités d'azote qu'elles peuvent libérer, les espèces étudiées se sont montrées prometteuses pour les pratiques agroforestières d'amélioration des jachères.

\section{REMERCIEMENTS}

Les auteurs remercient le CIRAD et l'IRD (France) pour le financement des activités de recherche et l'appui scientifique appréciable.

\section{BIBLIOGRAPHIE}

Agbahungba A.G., 2007. Dynamique de la matière organique d'Acacia auriculaeformis (cunn. A.) ex benth.et influence de la fertilisation organique et minérale dans les systèmes agroforestiers d'Acacia à base de maïs : Zea mays l.(Poaceae), dans le Sud du Bénin.

Aerts R., 1996. Nutrient resorption from senescing leaves of perennials: are there general patterns? Journal of Ecology 84:597-608

Ballé P. et Ouattara N.,1986. Essai d'enrichissement des jachères. Présentation et mise en place. Rapport CTFT, Abidjan, Côte d'Ivoire. 5 p.

Bernhard-Reversat F., Diangana D., Tsata M. (1993). Biomasse, minéralomasse et productivité en plantation d'Acacia mangium et Acacia auriculiformis au Congo. Bois et Forêt des Tropiques $n^{\circ} 238$ pp. 35-43.

Boyer J., 1982. Les sols ferralitiques. Tome X. Facteurs de fertilité et utilisation des sols. Edition ORSTOM, $n^{\circ}$ 52, Paris, France. 384 p.

Chiharu M., Yukihiro C, Takeshi T., 2007. Seasonal and spatial variations in leaf nitrogen content and resorption in a Quercus serrata canopy. Tree Physiology 27:63-70

Clément J., 1970. Rapport d'activités de la station d'expérimentation forestière d'Oumé de 1965 à 1969. CTFT Abidjan, Côte d'Ivoire. 93 p.

De Rouw A. (1993). Influence du raccourcissement de la jachère sur l'enherbement et la conduite des systèmes de cultures en zone forestière. In Floret $\mathrm{Ch}$ et Serpantié Georges (ed.). La jachère en Afrique de l'Ouest. pp 257-266.

De Tafin G., Zakra N., Pomier M., Braconnier S., Weawer R.W., 1991. Recherche d'un système cultural stabilisé associant le cocotier à des arbres fixateurs d'azote. Oléagineux. vol. 46, $n^{\circ} 12, \mathrm{pp} 489-500$.

Dommergues V. Duhoux E. et Diem H.G. (1999). Les arbres fixateurs d'azote. Caractéristiques fondamentales et rôle dans l'aménagement des écosystèmes méditerranéens et tropicaux. Edition CIRAD-IRD FAO. 494 p.

Dupuy B. et N'guessan K. A., 1990. Utilisation des acacias pour régénérer les anciennes cocoteraies. Bois et Forêts des Tropiques, 230 : 15-29.

Dupuy B. et N'guessan K.A. (1991). Sylviculture d'Acacia mangium en Basse Côte d'Ivoire. Bois et Forêts des Tropiques, $N^{\circ} 225$, pp 2432.

Floret Ch. et Pontanier R., 2000. La jachère en Afrique tropicale. Rôles, aménagements, alternatives. Actes du Séminaire International, Dakar, Sénégal, du 13-16 avril 1999. Vol. 1, 777 p.

Floret Ch. et Serpantié G., 1993. La jachère en Afrique de l'Ouest. Actes de l'Atelier International du 02 au 05 décembre 1991. Montpellier, France, $494 \mathrm{p}$.

Gnahoua G.M., 2004. Contribution des légumineuses à la régénération des jachères : Intérêts et limites des arbres fixateurs d'azote en zone forestière de Côte d'Ivoire. Thèse de DocteurIngénieur en Sciences Agronomiques. Université de Cocody-Abidjan, Côte d'Ivoire, $142 \mathrm{p}$.

Gona-Tchimbakala, J., Ndondou-Hockemba M., Kokolo A., Mboussou-Kimbangou A.N.S., 2005. Variations des apports de litière et d'éléments minéraux dans les plantations de Limba (Terminalia superba). TROPICULTURA, 2005, 23, 1, 53-59

Gutterridge C., 1992. Evaluation of leaf of the range of tree legumes as a source of nitrogen for crop growth. Expl. Agricult., 28 p. 195-202.

Jouve $P_{\text {., }}$ 2001. Jachère et systèmes agraires en Afrique subsaharienne. In Floret $\mathrm{Ch}$. et Pontanier R., Eds. La jachère en Afrique tropicale :Rôles, aménagements, alternatives. Actes du Séminaire International, Dakar, Sénégal, du 13-16 avril 1999. Vol. 2, pp 1-20. 
Moreau R. (1993). Influence de la mise en culture et de la jachère forestière sur l'évolution des sols tropicaux. In la jachère en Afrique de l'Ouest. Actes de l'Atelier International du 02 au 05 décembre 1991. Montpellier, France pp. 245256.

National Academy of Sciences., 1983. Mangium and other fast-growing Acacias for the humid Tropics. National Academy Press. Washington D.C.USA, $62 \mathrm{p}$.

Oliver R. et Ganry F. (1994). Étude des modifications de fertilité induite par une jachère arborée. Cas de la zone forestière de Côte d'Ivoire. Compte rendu d'une recherche financée par le Ministère de la Recherche et de la Technologie. CIRAD Montpellier, France, 27 pages.

Oualou K., 1989. Agroforesterie et développement rural intégré en Côte d'Ivoire : le cas de la région
d'Oumé. Mémoire de Master of Sciences. Université Laval, Canada, 191 p.

Peltier R. et Eyog-Matig O. (1988). Les essais d'agroforesterie du Nord Cameroun Bois et Forêts des Tropiques. $\mathrm{N}^{\circ} 217$, pp 3-23.

Schröt G., Kolbe D., Pitty B. and Zech W., 1995. Searching for criteria of the selection of efficient tree species for fallow improvement, with special reference to carbon and nitrogen. Fertilizer Research, 42 : 297-314.

Zakra A.N., 1997. Contribution à l'étude de la restauration et du maintien de la fertilité des sables quaternaires du littoral ivoirien : Cas de l'utilisation d'arbres fixateurs biologiques d'azote comme plantes associatives avec les cocotiers. Thèse de Docteur-Ingénieur. Université d'Abidjan, Côte d'Ivoire, 152 p + annexes. 